\title{
The Impact of Tourniquet Release Time on Wound Healing in Patients Undergoing Tibia Fracture Plating Surgery
}

\author{
Gholamhossein Mahmoudirad, ${ }^{1}$ Reza Bahador, ${ }^{2}$ and Maryam Khodabakhshi ${ }^{3,}$ \\ ${ }^{1}$ Deparetment of Nursing, Faculty of Nursing and Midwifery, Birjand University of Medical Sciences, Birjand, Iran \\ ${ }^{2}$ Department of Orthopedic Surgery, Faculty of Medicine, Birjand University of Medical Sciences, Birjand, Iran \\ ${ }^{3}$ Faculty of Nursing and Midwifery, Birjand University of Medical Sciences, Birjand, Iran \\ "Corresponding author: Maryam Khodabakhshi, M.Sc. Student of Medical-Surgical Nursing Education, Faculty of Nursing and Midwifery, Birjand University of Medical \\ Sciences, Birjand, Iran. Tel: +98-9371638928, E-mail: khodabakhshim2013@gmail.com
}

Received 2016 January 11; Revised 2016 February 27; Accepted 2016 March 13

\begin{abstract}
Background: The use of tourniquet is very common in orthopedic surgeries. By obstructing blood flow in the limb, tourniquet may result in muscle ischemia and skin flap hypoxia. This study aimed at determining and comparing the effects of tourniquet release time on wound healing in patients undergoing tibia fracture plating surgery.

Methods: This study was a randomized clinical trial, wherein 40 patients with acute extra-articular tibia fractures were randomly assigned to 2 groups of A (releasing the tourniquet after fracture fixation and before wound closure) and B (releasing the tourniquet after wound closure and application of compression dressing). Duration of surgery in each group was recorded and independent $t$ test was utilized to compare the 2 groups. The wound healing rate was investigated in the patients 24 hours and 14 days after surgery using the Redness, Oedema, Ecchymosis, Discharge, Approximation (REEDA) scale. In this tool, o represents "lack of the variable" and 3 indicates "maximum variable score". The scores in this scale range from 0 to 15 . The Mann-Whitney test was used in order to compare the wound healing rates between the 2 groups.

Results: There was no significant difference between the 2 groups concerning the average duration of surgery. Wound redness, edema, ecchymosis, discharge, approximation, and the general condition of wound healing showed no significant difference in the 2 groups 24 hours after surgery, while there was a significant difference 14 days after surgery with the aforementioned parameters being greater in group B than group A.

Conclusions: The results showed that releasing the tourniquet before wound closure in group A led to improved wound healing. Wounds need oxygen for restoration and prevention from infections. As the use of tourniquet occludes blood flow to the limb for a while, it can result in increased wound hypoxia after surgery and delayed healing process; hence, less tourniquet time is more desirable for oxygenation of tissues and wound dryness.
\end{abstract}

Keywords: Tourniquet, Tibia Fracture, Internal Fixation of Fractures, Wound Healing, Infection

\section{Background}

The use of tourniquet is very common in orthopedic surgeries. Tourniquet is used to prevent bleeding during surgery and create a blood-free environment for the convenience of the surgeon; in other words, tourniquet makes the surgery more convenient (1). Generally, tourniquets can be pneumatic (using air pump) or Esmarch. Nowadays, most tourniquets are of the pneumatic type(2). Pneumatic tourniquets, first proposed in 1904 by Cashing, contain cuffs, which encircle the limb and are inflated with compressed gas $(3,4)$. Tourniquets have been used for a century by surgeons for operations on lower limbs, with higher pressure than systolic blood pressure (5).

Tourniquets are widely used by orthopedic surgeons as they have significant benefits such as reduced intraoperative bleeding, clean and bloodless surgical field, better view of the surgical site and anatomical structures, im- proved cementing via providing clean and dry bone surfaces, reduced operation time and therefore lower risks of infections (6). However, similar to all other surgical interventions, the use of tourniquet may have its own complications (4). Neurovascular damage, limb pain, deep vein thrombosis (DVT), edema, cardiorespiratory effects, pulmonary embolism, acute pulmonary edema, cardiac arrest, increased postoperative bleeding, muscle ischemia, rhabdomyolysis, skin flap hypoxia, and delayed wound healing are some of the reported complications of tourniquets (6).

The most prevalent methods of using a tourniquet include its release before wound closure and hemostasis, or after wound closure and application of compression dressing (7). Some researchers believe that releasing the tourniquet before wound closure and hemostasis is a credible and sensible approach, in which the patients experience less postoperative pain (8), less blood loss (9), improved 
and rapid performance $(8,9)$, and reduced risk of complications (10).

A common application of tourniquet is in leg fracture fixation surgeries. Leg fracture is one of the most common causes of referring to orthopedic centers and hospitals (11). Among leg fractures, the tibial bone fracture is one of the most common caused by urban trauma, which usually follows accidents with motor vehicles (12).

Based on previous studies, tibia is the most common fracture area in individuals under 50 years of age (13). According to the US National center for health statistics (NCHS), about 490000 cases of tibia fractures occur annually in the United States. The annual incidence of this kind of fracture is 1 in every 2 thousand individuals. In the past, orthopedic surgeons tended to treat these fractures with nonsurgical approaches. Nowadays, there is a greater tendency toward surgical treatment of such fractures, including plate and screw fixation with intramedullary nail and external fixation (14).

Due to the wide gap of the surgical site and periosteum detachment, plating surgery may be accompanied by bleeding and increased risk of infection (15). Tourniquet is usually used to reduce bleeding during plating surgery (1). Because tourniquet occludes the blood flow to the limb for a while, it can improve wound healing. Wound healing is especially important in tibia bone. In their 10-year experience of the surgical treatment of tibia fractures, Rommens and Schmit-Neuerburg observed that minor wound complications, relative necrosis of the wound edges, and postoperative hematoma occurred in $12.7 \%$, 9.5\%, and 5.5\% of the patients with closed fractures, and $10.9 \%, 7.1 \%$, and $3.9 \%$ of patients with open fractures of the tibia, respectively (16). In their study of the role of tourniquet in wound infection after fibula fracture surgery, Maffulli et al. (1993) found that the use of tourniquet increased the possibility of infection (17). In contrast, Choksy et al. (2006), who investigated the effect of tourniquet in transtibial amputation, reported no difference in the wound healing rate and wound breakdown between tourniquet and nontourniquet groups (18).

According to Boack (2011), timing of the tourniquet has a direct impact on wound complications in Total Ankle Replacement (TAR), so that the patients with 30 minutes or more of tourniquet time had 5.1\% greater wound complications (19).

Since the studies on the effect of tourniquet on wound healing showed inconsistent results, and only a few studies have evaluated the impact of tourniquet time in tibia surgeries, the present study aimed at determining and comparing the effects of tourniquet time on wound healing in internal fixation surgeries of tibia fractures in order to promote knowledge of physicians, operating room techni- cians, and nurses in this regard.

\section{Methods}

This was a randomized, double-blind controlled clinical trial, wherein a total of 40 eligible participants (20 in each group) were selected from candidates of tibia fracture plating surgery in the orthopedic department of Imam Reza hospital of Birjand, eastern Iran in 2016. The candidates for tibia fracture plating surgery were visited the day before. Sufficient information about the implementation of the plan was provided for all participants. After signing the informed consent form, eligible participants were selected by the convenience sampling method. Immediately before the surgery, the patients were randomly assigned into 2 groups of $A$ and $B$ by permuted block randomization method.

The patients with the following characteristics entered the study: having tibia fracture, age of above 18 and below 55 years old, having closed fracture, which was not in the knee joint, undergoing surgery in the first week after the fracture, lack of history of surgery on the limb, lack of fractures in other limbs, lack of chest, head or abdominal trauma, lack of neurovascular damage and compartment syndrome; lack of coagulation difficulties, peripheral vascular disease, peripheral neuropathy and DVT in the limb before the operation; lack of consumption of steroidal and non-steroidal anti-inflammatory drugs and anti-coagulation drugs, chemotherapy, cigarette and drug abuse, body mass index of less than 30 , systolic blood pressure of less than $200 \mathrm{mmHg}$, and having class I and II (ASA class I: healthy patients, no organic biochemical or psychological disease; ASA class II: the patient with mild systemic disease that does not affect the routine activity) American society of anesthesiology (ASA) criterion. Patients with more than 90 minutes of tourniquet time, those that needed to be operated again due to any reason, those who were not willing to cooperate in any phase of the research, and those who passed away were excluded from the study.

A cuff with a length of $75 \mathrm{~cm}$ and a width of $15 \mathrm{~cm}$ was placed on the most muscular area of the patients' femur after putting 3 layers of Webril padding. The tourniquet pressure was adjusted as $150 \mathrm{mmHg}$ higher than the systolic blood pressure of each patient. Tourniquet pressure levels and blood pressure were recorded for all patients. After prepping and draping, the limb blood drainage was performed by raising the limb for 3 minutes. The tourniquet cuff was then inflated according to the adjusted pressure. All patients underwent open reduction and internal fixation of fractures of the tibia with plate and screws by a surgeon using standard techniques and implants. In group A, the tourniquet was released after completion of 
fracture fixation and before wound closure. In group B, the tourniquet was released after wound closure and application of compression dressing. The utilized tourniquet was of pneumatic type with MICROBACE brand (I. type BFclass, manufactured by RAEENSANAT Co., Iran). In all patients, hemostasis with electrocauter was performed by Matin electrocautery device (MEG1 model, manufactured by KAVANDISH SYSTEM Co., Iran). The subcutaneous tissue was re-approximated with Supabon sutures and the skin was closed with nylon sutures. In both groups, the limb was bandaged with Webril and stretch bandages. The Hemovac drain was placed on the surgical site in order to control the bleeding and discharge, and it was removed 24 hours after surgery.

For data collection, the demographic form and redness, edema, ecchymosis, discharge, approximation (REEDA) scale were used. Follow up of the wound healing process was performed 24 hours and 14 days after surgery, based on REEDA scale for assessment of the wounds. This tool has 5 criteria of redness, edema, ecchymosis, discharge, and approximation, each being assigned a score of 0 to 3: 0 represents a lack of the variable and 3 is the maximum score for presence of the variable (Table 1). The validity and reliability of this tool were confirmed in previous studies (20). The follow-up of wound healing process within 24 hours after surgery was performed by the examiner, who visited the hospitalized patients. In order to evaluate the wound healing process 14 days after surgery, all patients received a special clinic card with a specified date and time on it for referring to the clinic. Also, the day before the visit, every patient was reminded of the date by a phone call. It should be noted that the evaluators were not aware of each patient's group and intervention type.

This study received medical code of ethics (Ir.bums.1394.84) from the ethics committee of Birjand University of Medical Sciences, and was registered in the Iranian registry of clinical trials under the following code IRCT2015092824252N1. After completion of the implementation phases of the study, data were coded and analyzed in the SPSS V-22 statistical software. After ensuring the accuracy of data entry, descriptive statistics was used for preparation of the frequency tables, and in order to determine the relationship between quantitative variables, the independent $t$ test was utilized. The difference between the intergroup means was calculated by independent $t$ test (for normality of the data) and Mann-Whitney U test (for abnormality of the data) and the difference between the intragroup means was calculated by paired $t$ test. Spearman's correlation coefficient and univariate GLM model were also used for data analysis. In all of the tests, the confidence coefficient was estimated as 95\% and the significance level as 0.05 .

\section{Results}

The present study was carried out on 40 patients with acute extra-articular tibia fracture, who were randomized to 2 groups. The 2 groups were similar with respect to their age, gender, and other demographic characteristics, and there was no statistically significant difference between them (Table 1).

Results of the independent t test showed that there was no significant difference in the average tourniquet time and duration of surgery between the 2 groups of A and $B$ $(P>0.05)$. The average duration of surgery in group $A$ was approximately 13 minutes more than group B, yet, no significant difference was ultimately observed between the 2 groups $(\mathrm{P}=0.107)$. The Mann-Whitney test showed that the amount of wound redness, edema, ecchymosis, discharge, approximation, and the general condition of wound healing had no significant difference in the 2 groups of A and B, 24 hours after surgery (Table 2), while there existed a significant difference between the 2 groups 14 days after surgery and the foregoing amounts in group B were greater than group A (Table 3).

Based on the results of the independent $t$ test, 14 days after surgery the overall score of wound healing was 2.05 \pm 0.3 in group A and $6.1 \pm 0.6$ in group $\mathrm{B}$, which revealed a statistically significant difference between the 2 groups ( $P$ $<0.001)$. The average changes of the total wound healing score in group B was more than group A, which indicates a significant difference between the 2 groups $(P<0.001)$ (Table 4).

According to the results of the GLM model, in the univariate state, no significant relationship was observed between tourniquet time and wound healing levels within 24 hours after surgery, yet, 14 days after surgery, there was a significant relationship between tourniquet time and wound edema, wound discharge, and the general condition of wound healing. Results of Tukey's Post Hoc test revealed that the average tourniquet time in patients with wound discharge score of 1 was more than patients with wound discharge score of 0 , presenting a significant difference. Moreover, the average tourniquet time in patients with wound ecchymosis score of 1 was more than patients with wound ecchymosis score of 0 , again presenting a significant difference.

Spearman's correlation coefficient revealed no significant correlation between wound healing and fracture area, surgery duration, tourniquet pressure, and age, yet, a correlation coefficient of about -0.37 was estimated between the body mass index and total wound healing score 14 days 
Table 1. REEDA Scale

\begin{tabular}{|c|c|c|c|c|c|}
\hline \multirow[t]{2}{*}{ Points } & \multicolumn{3}{|c|}{ Score } & \multicolumn{2}{|r|}{ Total } \\
\hline & Redness & Edema & Ecchymosis & Discharge & Approximation \\
\hline o & None & None & None & None & Close \\
\hline 1 & Within $0.25 \mathrm{~cm}$ of the incision bilaterally & less than $1 \mathrm{~cm}$ from incision & Within $0.25 \mathrm{~cm}$ bilaterally or $0.5 \mathrm{~cm}$ unilaterally & Serum & Skin separation $3 \mathrm{~mm}$ or less \\
\hline 2 & Within $0.5 \mathrm{~cm}$ of the incision bilaterally & between 1 and $2 \mathrm{~cm}$ from the incision & $\begin{array}{l}\text { Between } 0.25 \mathrm{~cm} \text { and } 1 \mathrm{~cm} \text { bilaterally or between } 0.5 \\
\text { and } 2 \mathrm{~cm} \text { unilaterally }\end{array}$ & Serosan-guinous & Skin and subcutaneous fat separation \\
\hline 3 & Beyond $0.5 \mathrm{~cm}$ of the incision bilaterally & greater than $2 \mathrm{~cm}$ from incision & Greater than $1 \mathrm{~cm}$ bilaterally or $2 \mathrm{~cm}$ unilaterally & Bloody, purulent & Skin, subcutaneous fat and fascial layer separation \\
\hline
\end{tabular}

Table 2. Distribution of Demographic and Clinical Variables in the Study Groups ${ }^{\mathrm{a}}$

\begin{tabular}{|c|c|c|c|c|c|c|c|c|c|c|c|c|}
\hline \multirow[t]{2}{*}{ Group } & \multirow[t]{2}{*}{ Age } & \multicolumn{2}{|c|}{ Gender } & \multicolumn{2}{|c|}{ Limb } & \multicolumn{3}{|c|}{ Anatomic Site of Fracture } & \multirow[t]{2}{*}{ Systolic Blood Pressure } & \multirow[t]{2}{*}{ Tourniquet Pressure } & \multicolumn{2}{|c|}{ Time, min } \\
\hline & & Female & Male & Right Leg & Left Leg & Proximal & Shaft & Distal & & & Tourniquet & Surgery \\
\hline A & $31.85 \pm 2.65$ & $3(15)$ & $17(85)$ & $10(50)$ & $10(50)$ & $1(5)$ & $12(60)$ & $7(35)$ & $119.7 \pm 4.7$ & $269.7 \pm 4.7$ & $53.2 \pm 4.7$ & $77.5 \pm 5.5$ \\
\hline B & $29.35 \pm 7$ & $3(15)$ & $17(85)$ & $8(40)$ & $12(60)$ & $1(5)$ & $13(65)$ & $6(30)$ & $118.2 \pm 2.4$ & $268.8 \pm 2.4$ & $55.5 \pm 4.4$ & $64.7 \pm 5.4$ \\
\hline P Value & 0.932 & \multicolumn{2}{|c|}{0.999} & \multicolumn{2}{|c|}{0.525} & \multicolumn{2}{|c|}{0.943} & 0.781 & 0.859 & 0.730 & 0.107 & \\
\hline Statistical Test & \multicolumn{8}{|c|}{ Fisher's Test } & \multicolumn{4}{|c|}{ Independent T-Tests } \\
\hline
\end{tabular}

Table 3. Distribution of Wound Healing Variables in the Study Groups

\begin{tabular}{|c|c|c|c|c|c|c|}
\hline \multirow[t]{3}{*}{ Variable } & \multicolumn{3}{|c|}{24 Hours After Surgery } & \multicolumn{3}{|c|}{14 Days After Surgery } \\
\hline & \multicolumn{2}{|c|}{ Groups } & \multirow[t]{2}{*}{ Mann-Whitney test } & \multicolumn{2}{|c|}{ Groups } & \multirow[t]{2}{*}{ Mann-Whitney test } \\
\hline & $\mathbf{A}$ & B & & $\mathbf{A}$ & B & \\
\hline Redness & $0.4(0.1)$ & $0.2(0.1)$ & 0.221 & $1.0(0.2)$ & $1.7(0.1)$ & 0.015 \\
\hline Edema & $0.5(0.1)$ & $0.7(0.1)$ & 0.192 & $0.8(0.1)$ & $1.5(0.1)$ & 0.007 \\
\hline Ecchymosis & $0.8(0.1)$ & $0.4(0.1)$ & 0.056 & $0.1(0.06)$ & $1.0(0.2)$ & 0.001 \\
\hline Discharge & $0.5(0.1)$ & $0.6(0.1)$ & 0.620 & $0.1(0.06)$ & $(0.2) 1.2$ & $<0.001$ \\
\hline Approximation & 0 & $0.05(0.05)$ & 0.330 & $0.05(0.05)$ & $0.6(0.1)$ & 0.002 \\
\hline Total score & $2.2(0.3)$ & $2.1(0.4)$ & 0.718 & $2.05(0.3)$ & $6.1(0.6)$ & $<0.001$ \\
\hline
\end{tabular}

Table 4. The Average Total Score of Wound Healing in the Study Groups

\begin{tabular}{lcccc}
\hline Group & Total Score of Wound Healing After 24 Hours & Total Score of Wound Healing After 14 Days & Paired t Test & Average Changes \\
\hline A & $2.2 \pm 0.3$ & $2.05 \pm 0.3$ & $-0.1 \pm 0.5$ & 0.943 \\
B & $2.1 \pm 0.4$ & $6.1 \pm 0.6$ & $4.1 \pm 0.7$ & 0.001 \\
Independent T-tests & 0.718 & $<0.001$ & - \\
\hline
\end{tabular}

after surgery, which indicates an inverse relationship between the body mass index (BMI) and total wound healing score $(\mathrm{P}=0.019)$.

\section{Discussion}

This study aimed at determining and comparing the effects of tourniquet release time on wound healing in patients undergoing tibia fracture plating surgery. The results showed that group A patients, whose tourniquet was released before wound closure, experienced better wound healing procedures compared with group B patients, whose tourniquet was released after wound closure and application of compression dressing. Twentyfour hours after surgery, there was no significant difference between the 2 groups of A and B with respect to the amount of wound redness, edema, ecchymosis, discharge, approximation, and the general condition of wound healing; however, 14 days after surgery, there was a significant difference between the 2 groups and the foregoing 
amounts were greater in group B than group A.

A few studies have evaluated the impact of tourniquet release time on wound healing rates in orthopedic surgeries of the tibial bone. However, various animal and human studies have investigated the use of tourniquet in tibia fractures. Although, these studies have not specifically dealt with wound healing rates as done in the present study, they noted some of the wound complications. In their study of the effect of tourniquet in plating of tibia fractures, Anvar Salam et al. (1991) reported 6 cases of erythema and wound induration in the tourniquet group, while neither of these complications were observed in the non-tourniquet group (21); in contrast, Choksy et al. (2006), who investigated the effect of tourniquet in transtibial amputation, reported no difference in wound healing and wound breakdown rates between the tourniquet and non-tourniquet groups (18).

Olivercrona et al. (2012) studied the effects of tourniquet pressure on wound complications in knee arthroplasty, based on limb occlusion pressure (LOP) and systolic blood pressure (SBP) methods. At the time of discharge from the hospital, 47 patients (30\%) had surgery wound complications such as blisters, wound discharge or signs of infection, 40 of which had experienced higher than $225 \mathrm{mmHg}$ cuff pressure during the surgery. During the 2-month follow up after surgery, 7 patients (4\%) had wound infection and 9 patients (6\%) suffered from delayed wound healing. Four of these patients had deep wound infection and needed repeated hospitalization and surgery (22). In our study, there was no statistically significant difference between the 2 groups in the mean tourniquet pressure, and no significant correlation was observed between wound healing and tourniquet pressure at any of the mentioned times.

Certain studies have measured the amount of wound infection after surgery with tourniquet $(1,17,23)$. Wound infection is an important criterion for wound healing, so that increased incidence of infection can be associated with a decrease in wound healing. In this regard, these studies can be compared with the present study. Saeid et al. (2010) studied the effect of tourniquet in tibia fracture plating surgery, and reported no statistically significant difference in the overall "infection" items between the 2 tourniquet and non-tourniquet groups (1). In their meta-analysis of the efficacy of tourniquet on ankle trauma surgery, Jiang et al. (2015) reported no significant difference between the tourniquet and non-tourniquet groups, regarding the amount of postoperative infection (23). In their study on the role of tourniquet in wound infection after fibula fracture surgery, Maffulli et al. (1993) found that the use of tourniquet increases the possibility of infection (17).

Only in total knee arthroplasty studies, researchers have investigated the impact of tourniquet release time, similar to the present study. In their systematic review and meta-analysis of the "effects of the timing of tourniquet release in cemented total knee arthroplasty" Zhang et al. (2014) indicated that releasing the tourniquet before wound closure could decrease the risk of major complications, such as infection, wound dehiscence, and hematoma with the need for drainage or debridement ( $P$ $<0.049$ ) (7). In their meta-analysis study, F. Zan et al. (2014) reported that releasing the tourniquet after wound closure increased the risk of minor complications such as erythema, necrosis of wound edges, cellulitis, superficial infection, discharge, and edema as well as major complications, including wound dehiscence, hematoma, and deep infection with the need for drainage or debridement $(\mathrm{P}<$ 0.05) (24). Kashif Abbas et al. (2013) reported in their study titled "effect of early release of tourniquet in total knee arthroplasty" that in Group B with tourniquet release after application of compression dressing, 4 patients (3.1\%) had wound complications, 3 of whom had trivial ones and 1 of whom needed a second surgery, yet, no such cases were observed in group $\mathrm{A}(\mathrm{P}=0.1192)$. According to the authors of that article, hematoma at the surgical site and closure of the tissues without homeostasis may be the cause of increased wound complications (25). Christodoulou et al. (2004) asserted that even though wound complications and hematoma were greater in group B, no significant difference was observed between the 2 groups (26). This may be due to the short ischemia period resulting from tourniquet in group A and blood opportunity for rushing to the edges of the wound before suturing and application of compression dressing. In group B, suturing the skin and subcutaneous tissue and application of compression dressing before releasing the tourniquet could completely inhibit the blood flow to the tissues by collapsing and occluding the arteries, consequently delaying the wound healing process due to the lack of oxygen and other nutrients. The wound needs oxygen for restoration and prevention from infection. While there are various reasons for delayed wound healing, oxygen delivery is the limiting factor, and delayed healing must be reduced by minimizing hypoxia. Tourniquet is supposed to be used for providing a blood-free surgical field, and it is an obvious cause of hypoxia during the surgery and a significant risk factor for hypoxia after surgery (27). When the tourniquet is inflated and the blood flow stops, small distal vessels are occluded by cellular debris and vascular contraction. This occlusion is not resolved in the early revascularization and, therefore, re-oxygenation of the tissue is prohibited, leading to the increased possibility of damaged tissues around the wound (28). Butt et al. (2011) believed that the migration of macrophages and fibroblasts to the wound is secondary 
to the formed oxygen gradient between the capillaries of the wound edges. This means that if the wound edges are hypoxic, angiogenesis and the migration of macrophages and fibroblasts, and consequently cell response to restoration are inhibited. Accordingly, lesser tourniquet times are more beneficial for oxygenation of the tissues and wound healing (29). Therefore, the use of tourniquet can increase wound hypoxia after surgery, particularly at higher pressures, and this can affect wound healing and may increase wound infection.

From the point of view of some researchers, hematoma at the surgical site and wound closure without homeostasis may be responsible for increased wound complications (25). In contrast, several studies have suggested that releasing the tourniquet before wounds closure increases the required time for tissue homeostasis, duration of surgery and anesthesia (30-32), and thus increases the possibility of postoperative infection. In the present study, the mean duration of surgery did not have a significant difference between the 2 groups of $A$ and $B$, and was in line with the results of the study by Yu Fan (2014) for knee arthroplasty (33).

In the current study, a significant relationship was observed between tourniquet time and the amount of wound edema, discharge, and the general condition of wound healing 14 days after surgery. Butt et al. (2010) also reported a significant relationship between the increase in tourniquet time and wound discharge after total knee arthroplasty (29), which is consistent with the results of this study. Several studies have approved that prolonged wound discharge increases the risk of infection (1317). Wound discharge is generally inevitable in the process of treatment, yet, if it continues for a long time, it will turn to a risk factor for infection and an increase in costs and length of hospital stay (34). Therefore, it is more efficient to release the tourniquet before wound closure and reduce the tourniquet time in order to reduce the possibility of this complication and therefore the risk of wound infection.

Despite the same drug treatment (antibiotics, analgesics, and vitamin c) in both groups, one of the limitations of this study was the lack of full control over the nutritional condition and the amount of physical mobility in different participants. Nevertheless, it was tried to somehow control these issues by randomized selection of people and presentation of identical training.

\subsection{Conclusion}

According to the results, the total score of wound healing with the indices of wound redness, edema, ecchymosis, discharge, and approximation in group A, whose tourniquet was released during the surgery and before wound closure was significantly lower 14 days after surgery, and therefore the wound healing levels were better in this group. The potential cause can be quicker blood supply to the limb and decreased tissue hypoxia. Theoretically, due to the fact that the use of tourniquet occludes blood flow to the limb for a while, it can lead to infection, and this is of greater importance in tibia surgeries. Since a few studies have evaluated the impact of tourniquet on wound healing in tibia fracture surgeries, it is suggested that more research must be conducted in this regard.

\section{References}

1. Saied A, Zyaei A. Tourniquet use during plating of acute extraarticular tibial fractures: effects on final results of the operation. J Trauma. 2010;69(6):E94-7. doi: 10.1097/TA.ob013e3181dbac93. [PubMed: 20571455].

2. Saied A, Ayatollahi Mousavi A, Arabnejad F, Ahmadzadeh Heshmati A. Tourniquet in surgery of the limbs: a review of history, types and complications. Iran Red Crescent Med J. 2015;17(2):e9588. doi:10.5812/ircmj.9588. [PubMed: 25793122].

3. Shemshaki H, Tehranfar AL, Saadati H, Jalalian M. Effects of pneumatic tourniquet on knee surgery. Australasian Med J. 2010:6882-8. doi: 10.4066/amj.2010.373.

4. Zhang Y, Li L, Wang J, Li ZH, Shi ZJ. Do patients benefit from tourniquet in arthroscopic surgeries of the knee?. Knee Surg Sports Traumatol Arthrosc. 2013;21(5):1125-30. doi: 10.1007/s00167-012-2094-3. [PubMed: 22699853].

5. Lohmann-Jensen R, Holsgaard-Larsen A, Emmeluth C, Overgaard $S$, Jensen $C$. The efficacy of tourniquet assisted total knee arthroplasty on patient-reported and performance-based physical function: a randomized controlled trial protocol. BMC Musculoskelet Disord. 2014;15:110. doi: 10.1186/1471-2474-15-110. [PubMed: 24678741].

6. Whitehead DJ, MacDonald SJ. TKA sans tourniquet: let it bleed: opposes. Orthopedics. 2011;34(9):e497-9. doi: 10.3928/01477447-2011071444. [PubMed: 21902143].

7. Zhang W, Liu A, Hu D, Tan Y, Al-Aidaros M, Pan Z. Effects of the timing of tourniquet release in cemented total knee arthroplasty: a systematic review and meta-analysis of randomized controlled trials. $J$ Orthop Surg Res. 2014;9:125. doi: 10.1186/s13018-014-0125-0. [PubMed: 25467223].

8. Barwell J, Anderson G, Hassan A, Rawlings I. The effects of early tourniquet release during total knee arthroplasty: a prospective randomized double-blind study. J Bone Joint Surg Br. 1997;79(2):265-8. doi: 10.1302/0301-620X.79B2.7191. [PubMed: 9119854].

9. Kvederas G, Porvaneckas N, Andrijauskas A, Svensen $\mathrm{CH}$, Ivaskevicius J, Mazunaitis J, et al. A randomized double-blind clinical trial of tourniquet application strategies for total knee arthroplasty. Knee Surg Sports Traumatol Arthrosc. 2013;21(12):2790-9. doi:10.1007/s00167012-2221-1. [PubMed: 23052115].

10. Rama KR, Apsingi S, Poovali S, Jetti A. Timing of tourniquet release in knee arthroplasty. Meta-analysis of randomized, controlled trials. J Bone Joint Surg Am. 2007;89(4):699-705. doi: 10.2106/JBJS.F.00497. [PubMed: 17403789].

11. Madadi F, Valavi N, Ansari K, Ghaemmaghami S. The frequency of tibia fractures in adults(1996-2003) [in Persian]. Iranian J Orthop Surg. 2005;3(2):42-7.

12. Sepehri B, Rameshi M. Effect of placement and material properties of tibial plate on stress pattern at fractured site [In Persian]. Modares Mech Eng. 2015;14(11):159-65. 
13. Janmohammadi N, Montazeri M, Akbarnezhad E. The Epidemiology of extremity fractures in trauma patients of Shahid Beheshti hospital, Babol, 2001-2006 [In Persian]. Iranian Emergen Med. 2014;1(1):34-9.

14. Husseinpoor Feizi H, Seddighi A, Sadeghpoor A, Soleimanpoor J, Ganjpoorsales J, Neiazi M. The evaluation results of ender in tibial shaft fractures [In Persian]. Medi J Tabriz Univ Med Sci. 2006;28(2):49-53.

15. Sarafan N, Mahdinasab SAAH, Marashinezhad S, Momenian M. Treatment of open tibia fracture by unreamed tibial nailing [In Persian].J Orthop Surg. 2006;2(4):110-6.

16. Rommens P, Schmit-Neuerburg KP. Ten years of experience with the operative management of tibial shaft fractures. J Trauma. 1987;27(8):917-27. doi: 10.1097/00005373-198708000-00012. [PubMed: 3612870].

17. Maffulli N, Testa V, Capasso G. Use of a tourniquet in the internal fixation of fractures of the distal part of the fibula. A prospective, randomized trial. J Bone Joint Surg Am. 1993;75(5):700-3. doi: 10.2106/00004623-199305000-00009. [PubMed: 8501085].

18. Choksy SA, Lee Chong P, Smith C, Ireland M, Beard J. A randomised controlled trial of the use of a tourniquet to reduce blood loss during transtibial amputation for peripheral arterial disease. Eur J Vasc Endovasc Surg. 2006;31(6):646-50. doi: 10.1016/j.ejvs.2006.03.008. [PubMed: 16750790].

19. Boack DH. Study reveals higher rate of infection with increased tourniquet time for total ankle replacement. American Orthop Foot Ankle Soci. 2011:14-6.

20. Alvarenga MB, Francisco AA, de Oliveira SM, da Silva FM, Shimoda GT, Damiani LP. Episiotomy healing assessment: Redness, Oedema, Ecchymosis, Discharge, Approximation (REEDA) scale reliability. Rev Lat Am Enfermagem. 2015;23(1):162-8. doi: 10.1590/0104-1169.3633.2538. [PubMed: 25806645].

21. Salam AA, Eyres KS, Cleary J, el-Sayed HH. The use of a tourniquet when plating tibial fractures. J Bone Joint Surg Br. 1991;73(1):86-7. [PubMed: 1991784].

22. Olivecrona C, Ponzer S, Hamberg P, Blomfeldt R. Lower tourniquet cuff pressure reduces postoperative wound complications after total knee arthroplasty: a randomized controlled study of 164 patients. J Bone Joint Surg Am. 2012;94(24):2216-21. doi: 10.2106/JBJS.K.01492. [PubMed: 23318611].

23. Jiang X, Yu B, Qu W, He J. Meta-analysis on the efficacy of tourniquet on ankle trauma surgery. Eur J Med Res. 2013;18:55. doi: 10.1186/2047783X-18-55. [PubMed: 24325911].
24. Zan PF, Yang Y, Fu D, Yu X, Li GD. Releasing of tourniquet before wound closure or not in total knee arthroplasty: a meta-analysis of randomized controlled trials. J Arthroplasty. 2015;30(1):31-7. doi: 10.1016/j.arth.2014.07.034. [PubMed: 25175846].

25. Abbas K, Raza H, Umer M, Hafeez K. Effect of early release of tourniquet in total knee arthroplasty. J Coll Physicians Surg Pak. 2013;23(8):562-5. [PubMed: 23930872]

26. Christodoulou AG, Ploumis AL, Terzidis IP, Chantzidis P, Metsovitis SR, Nikiforos DG. The role of timing of tourniquet release and cementing on perioperative blood loss in total knee replacement. Knee. 2004;11(4):313-7. doi: 10.1016/j.knee.2003.09.005. [PubMed: 15261219].

27. Johnson DP. Infection after knee arthroplasty. Clinical studies of skin hypoxia and wound healing. Acta Orthop Scand Suppl. 1993;252:1-48. [PubMed: 8493866].

28. Clarke MT, Longstaff L, Edwards D, Rushton N. Tourniquet-induced wound hypoxia after total knee replacement. $J$ Bone Joint Surg Br. 2001;83(1):40-4. doi: 10.1302/0301-620X.83B1.10795. [PubMed: 11245536]

29. Butt U, Ahmad R, Aspros D, Bannister GC. Factors affecting wound ooze in total knee replacement. Ann R Coll Surg Engl. 2011;93(1):54-6. doi: 10.1308/003588410X12771863937124. [PubMed: 20836920].

30. Hernandez-Castanos DM, Ponce VV, Gil F. Release of ischaemia prior to wound closure in total knee arthroplasty: a better method?. Int Orthop. 2008;32(5):635-8. doi: 10.1007/s00264-007-0376-z. [PubMed: 17503044].

31. Hersekli MA, Akpinar S, Ozkoc G, Ozalay M, Uysal M, Cesur N, et al. The timing of tourniquet release and its influence on blood loss after total knee arthroplasty. Int Orthop. 2004;28(3):138-41. doi: 10.1007/s00264004-0550-5. [PubMed: 15024499].

32. Yavarikia A, Amjad GG, Davoudpour K. The influence of tourniquet use and timing of its release on blood loss in total knee arthroplasty. Pak J Biol Sci. 2010;13(5):249-52. doi: 10.3923/pjbs.2010.249.252. [PubMed: 20464949].

33. Fan Y, Jin J, Sun Z, Li W, Lin J, Weng X, et al. The limited use of a tourniquet during total knee arthroplasty: a randomized controlled trial. Knee. 2014;21(6):1263-8. doi: 10.1016/j.knee.2014.08.002. [PubMed: 25212990].

34. Saleh K, Olson M, Resig S, Bershadsky B, Kuskowski M, Gioe T, et al. Predictors of wound infection in hip and knee joint replacement: results from a 20 year surveillance program. JOrthop Res. 2002;20(3):506-15. doi: 10.1016/S0736-0266(01)00153-X. [PubMed: 12038624]. 